\title{
Based on Network Pharmacology to Explore the Active Ingredients and Molecular Targets of Zuojin Pill for the Treatment of Ulcerative Colitis
}

\author{
Ying Wei ( $\nabla$ weiyingtcm@163.com ) \\ Sichen Ren \\ Chengdu University of Traditional Chinese Medicine \\ Ruilin Wang \\ 5th Medical Center of Chinese PLA General Hospital \\ Manyi Jing \\ 5th Medical Center of Chinese PLA General Hospital \\ Honghong Liu \\ 5th Medical Center of Chinese PLA General Hospital \\ Min Wang \\ 5th Medical Center of Chinese PLA General Hospital

\section{Hongtao Song} \\ 900th Hospital of PLA \\ Yanling Zhao \\ 5th Medical Center of Chinese PLA General Hospital
}

Chengdu University of Traditional Chinese Medicine https://orcid.org/0000-0001-9671-913X

\section{Research}

Keywords: Zuojin Pill, Network pharmacology, ulcerative colitis, bioactive compounds, potential mechanisms

Posted Date: March 17th, 2021

DOI: https://doi.org/10.21203/rs.3.rs-317290/v1

License: (c) (i) This work is licensed under a Creative Commons Attribution 4.0 International License. Read Full License 


\section{Abstract}

Background: Zuojin Pill (ZJP), a classic prescription, has the potential to prevent ulcerative colitis (UC). However, the active component and mechanism of ZJP is still arcane.

Objective: This study aims to use a network pharmacology approach to find the bioactive compounds and potential action mechanisms of ZJP in the treatment of UC.

Methods: Firstly, the components and putative targets of ZJP were collected based on the herbal medicine target database, and a network containing the interaction between the putative targets of ZJP and the potential therapeutic targets of UC was established. Then topological parameters were calculated to identify the key targets in the network and the key targets were imported into David database to perform path enrichment analysis.

Results: 7 potential therapeutic components of ZJP and 26 key targets were obtained. These targets were related to signal transduction, response to drug, cellular response to lipopolysaccharide, MAPK cascade, inflammatory response, immune response, transcription from RNA polymerase II promoter, apoptotic process, regulation of sequence-specific DNA binding transcription factor activity and lipopolysaccharidemediated signaling pathway. Moreover, PI3K-Akt signaling pathway, MAPK signaling pathway and Toll-like receptor signaling pathway were predicted to participate in the treatment of UC, which directly regulated by 7 active components of ZJP. Quercetin and isorhamnetin have great development value in the treatment of UC. Moupinamide and palmidin A are of great value for exploration because of their safety and innovation.

Conclusion: ZJP mainly were directly involved in UC through inflammation and immune regulation by PI3K-Akt signaling pathway and MAPK signaling pathway.

\section{Introduction}

Ulcerative colitis (UC) is a chronic non-specific inflammatory disease. It is listed as a modern refractory disease by the World Health Organization because of its unclear etiology, high clinical recurrence rate and association with colon cancer ${ }^{[1]}$.In recent year, the incidence rate of UC has been increasing worldwide ${ }^{[2]}$. Aminosalicylic acids, hormones and immunosuppressants are mainly used in the treatment of UC, but there are obvious adverse reactions. For example, mesalazine is the first-line drug in the treatment of ulcerative colitis, and its long-term application can damage the liver and kidney function of patients ${ }^{[3]}$. Therefore, many patients with UC as well as physicians and researchers are increasingly considering complementary and alternative medicine options ${ }^{[4-5]}$.

Zuojin Pill (ZJP), a classic prescription for gastrointestinal diseases, has been widely used in the clinical application of gastrointestinal diseases in China since ancient times because of its safety and effectiveness. It composes of Coptis chinensis Franch. (Ranunculaceae, recorded in the Chinese Pharmacopoeia as Rhizoma Coptidis) and Evodia rutaecarpa (Juss.) Benth. (Rutaceae, recorded in the Chinese Pharmacopoeia as Fructus Evodiae) (6:1, g/g). In recent years, it has been reported that ZJP can inhibit the protein expression of PI3K and pAkt and regulate the crosstalk between intestinal flora and Treg cells, thus alleviating experimental colitis ${ }^{[6]}$. However, Chinese medicine compound has many components, targets and curative effects. Consequently, the potential active ingredients and molecular targets of ZJP on UC are hardly clarified from a single aspect. With the rapid development of bioinformatics, systems biology and polypharmacology, pharmacology method based on network pharmacology has been proved to be an effective mean to delving further into the active ingredients and targets of traditional Chinese medicine ${ }^{[7-9]}$.

Therefore, this study aims to use a comprehensive network pharmacology-based approach to investigate the potential effective components and molecular targets of ZJP in treating UC. The flowchart of the experimental procedures of our study was shown in Fig. 1.

\section{Materials And Methods}

\section{Chemical components of ZJP}

We searched Traditional Chinese Medicine Systems Pharmacology Database (TCMSP, http://lsp.nwu.edu.cn/tcmsp.php, updated on May 31, 2014), Chinese Academy of Sciences Chemistry Database (CASC, http://www.organchem.csdb.cn/scdb/main/slogin.asp, updated on December 31, 2019) and related literatures to collect the chemical components of the two herbs contained in ZJP using "Coptidis Rhizoma" and "Evodiae Fructus" as the queries. TCMSP is a unique systematic pharmacology database of Chinese herbal medicines, chemicals, targets and drug-target networks. CASC is one of the most comprehensive chemical databases in the world, which can provide chemical information of Chinese herbal medicine and natural products. 


\section{Candidate targets of ZJP}

The targets of chemical components in ZJP were mainly obtained from TCMSP databases. If the targets of chemical constituents reported in the literature are not included in the database, they are obtained through SwissTargetPrediction (http://www.swisstargetprediction.ch/).

\section{Establishment of target database for treating UC}

The known therapeutic targets of UC were acquired from DisGeNET database (http:// www.disgenet.org/) and OMIM database (https://omim.org/).After screening the target (score $\geq 0.01$ ) and removing the duplicate value, they were collected and used for data analysis serving as a target database for treating UC.

\section{Construction and analysis of ingredient-target-disease interactive network}

The ingredient-target-disease network was constructed by importing the active ingredients and potential targets into Cytoscape v3.7.1. Three topological parameters were used as the screening criteria to obtain hub network and key targets. (degree centrality $\geq 2 \times m e d i a n$ degree centrality; betweenness centrality $\geq$ median betweenness centrality; closeness centrality $\geq$ median closeness centrality).

\section{Construction and analysis of PPI network}

The protein-protein interactions (PPI) of each target were generated from STRING database (https://string-db.org/), and the interactions with a probabilistic association confidence score $\geq 0.9$ were selected in this study. All networks were constructed and analyzed by using Cytoscape v3.7.1.

\section{GO and KEGG pathway enrichment analysis}

Gene Ontology (GO) analysis and Kyoto Encyclopedia of Genes and Genomes (KEGG) pathway enrichment analysis were performed using the database for Annotation, Visualization and Integrated Discovery (DAVID) (https://david.ncifcrf.gov/). GO terms and KEGG pathways with $p$ value 0.05 were considered statistically significant.

\section{Construction and analysis of Component-Target-Pathway network}

Components-Targets-Pathways network was constructed and visualizatied via Cytoscape v3.7.1 to identify key and directly linked molecules and targets. The directly linked molecules were screened by Oral bioavailability $(\mathrm{OB})$ and Drug-likeness(DL)(OB $\geq 30 \%, \mathrm{DL} \geq 0.18)$.

\section{Toxicity prediction of components in ZJP}

ADMETlab (http://admet.scbdd.com) was a free tool database for the prediction of ADMET properties, and has been widely used in chemical and pharmaceutical fields. Each SMILES of molecule was input to obtain organ toxicity and other toxic properties.

\section{Results}

\section{Composite ingredients of ZJP}

A total of 156 chemical ingredients of the two herbal medicines in ZJP were collect from TCMSP, CASC and related literatures, including 32 ingredients in $R$. coptidis and 129 ingredients in E. rutaecarpa. There are 5 shared components: berberine, obacunone, quercetin, isovanillin, limonin (Fig.2A).

\section{Putative targets of components}

249 direct acting targets of Coptis chinensis and 357 direct acting targets of Evodia rutaecarpa were obtained. After removing repeated targets, 386 targets were obtained for subsequent analysis. And there were 220 overlapping targets between the two herbs (Fig.2B), which suggested that there might be interaction between Coptis chinensis and Evodia rutaecarpa in the treatment process.

\section{The known therapeutic targets of UC}

1458 targets of UC were obtained from DisGeNET database and 180 targets of UC obtained from OMIM database. A total of 1527 targets of UC were obtained after removing the repeats for subsequent analysis (Fig.2C). By comparing the components targets and the disease targets through the veen diagram, 146 targets for the treatment of UC were identified (Fig.2D). These targets may be the key proteins of ZJP in the treatment of UC. 
(A) Distribution of active compounds among the herbs(C: Coptidis Rhizoma ingredients; E: Evodiae Fructus ingredients). (B) Distribution of potential targets among the herbs(C: Coptidis Rhizoma targets; E: Evodiae Fructus targets; S: Shared targets). (C) Distribution of the known therapeutic targets of UC from two databases. (D) Distribution of ZJP targets and the disease targets.

\section{The ingredient-target-disease interactive network}

In order to comprehensively reveal the possible effective ingredients and potential targets of ZJP against UC, the ingredient-target-disease interactive network was established with 542 nodes and 1953 edges (Fig.3), containing 156 ingredients and 386 targets. Nodes have larger degree values and more edges, which play a more pivotal role in network regulation. Accordingly, the network of ingredients with common targets of drugs and the disease was constructed to display the interactions between active compounds and potential targets directly. There were 696 edges and 302 nodes containing 156 ingredients and 146 common targets of drugs as well as disease in the network. The 146 common targets were regarded as potential targets in ZJP for the treatment of UC (Fig.4). Degree represented the total number of routes connected to this node by other nodes in the network. The higher value, the more important corresponding ingredient or target was. Table 1 showed that the ingredient with the highest degree was quercetin (degree $=82)$. The following compounds were isorhamnetin $($ degree $=25)$, caffeine (degree $=20)$, (R)-Canadine (degree $=19$ ) and so on. In terms of targets, PTGS2 (degree = 95) was the most interacting ligands. The following targets were PTGS1 (degree $=73$ ), ADRB2 (degree $=48)$, NOS3 (degree $=27$ ) $\nabla C A L M 1$ (degree $=20)$ and so on. As a result, it was recognized that ZJP achieved the effect on UC mainly through the key targets above.

\section{Tab.1.The top 5 ingredients in the ingredients and common targets network}

\begin{tabular}{|llll|}
\hline Code name & Ingredient & Degree & Source \\
\hline CR3 & quercetin & 82 & Coptidis Rhizoma;Evodiae Fructus \\
R78 & isorhamnetin & 25 & Evodiae Fructus \\
R50 & caffeine & 20 & Evodiae Fructus \\
C6 & (R)-Canadine & 19 & Coptidis Rhizoma \\
C22 & Magnoflorine & 18 & Coptidis Rhizoma \\
\hline
\end{tabular}

\section{The PPI network}

To explore the mechanism of target genes of ZJP for the treatment of UC, the PPI network was constructed by STRING (confidence score $\geq 0.9$ ) with 125 nodes and 645 edges (Fig.5). Based on the topological parameters, we identified 26 key targets (Tab.2). The outcomes demonstrated that the target protein levels of JUN, MAPK1, TNF, PIK3CA and RELA were excessively crucial in ZJP for UC (Fig.6).

Tab.2. Hub genes in PPI network 


\begin{tabular}{|llll|}
\hline Gene name & Degree & Betweenness Centrality & closeness centrality \\
\hline JUN & 40 & 0.0866053 & 0.52765957 \\
\hline MAPK1 & 35 & 0.10522212 & 0.51239669 \\
\hline TNF & 33 & 0.06737271 & 0.49206349 \\
\hline PIK3CA & 32 & 0.09809998 & 0.49011858 \\
\hline RELA & 31 & 0.04010596 & 0.50612245 \\
\hline AKT1 & 31 & 0.07469638 & 0.49011858 \\
\hline TP53 & 30 & 0.06701158 & 0.49011858 \\
\hline FOS & 28 & 0.03619664 & 0.484375 \\
\hline SRC & 27 & 0.05381998 & 0.496 \\
\hline IL6 & 27 & 0.05383839 & 0.484375 \\
\hline MAPK14 & 26 & 0.02930984 & 0.47328244 \\
\hline MAPK8 & 24 & 0.0236753 & 0.46096654 \\
\hline VEGFA & 23 & 0.05077093 & 0.5 \\
\hline CTNNB1 & 22 & 0.03781139 & 0.46268657 \\
\hline EGFR & 22 & 0.05992162 & 0.48627451 \\
\hline SP1 & 21 & 0.03912481 & 0.46441948 \\
\hline ESR1 & 21 & 0.00980253 & 0.47148289 \\
\hline CXCL8 & 21 & 0.06791767 & 0.44604317 \\
\hline MYC & 20 & 0.00939055 & 0.46969697 \\
\hline IL1B & 19 & 0.01687947 & 0.46441948 \\
\hline JAK2 & 19 & 0.01407263 & 0.45925926 \\
\hline IL2 & 18 & 0.00668683 & 0.45588235 \\
\hline IL4 & 17 & 0.01066433 & 0.44765343 \\
\hline SMAD3 & 17 & 0.01141516 & 0.43055556 \\
\hline STAT1 & 16 & 0.01339176 & 0.44927536 \\
\hline NR3C1 & 16 & 0.00783583 & 0.44765343 \\
\hline
\end{tabular}

\section{GO and KEGG enrichment analysis}

To investigate the biological functions and pathway of the key targets of ZJP, the gene ontology (GO) biological process (BP) and KEGG were performed through the functional annotation tool of DAVID and Cytoscape3.7.1. The top $10 \mathrm{GO}$ terms and pathways were significantly enriched respectively (Fig.7, 8).

In UC, the BPs mainly regulated by ZJP were signal transduction, response to drug, cellular response to lipopolysaccharide, MAPK cascade, inflammatory response, immune response, transcription from RNA polymerase II promoter, apoptotic process, regulation of sequence-specific DNA binding transcription factor activity and lipopolysaccharide-mediated signaling pathway.

In UC, the pathways mainly regulated by ZJP were Pathways in cancer, Chagas disease, Hepatitis B, Toll-like receptor signaling pathway, Influenza A, Proteoglycans in cancer, MAPK signaling pathway, HTLV-I infection, PI3K-Akt signaling pathway and Prolactin signaling pathway (Tab.3). Among them, the changes of Toll-like receptor signaling pathway, MAPK signaling pathway and PI3K-Akt signaling pathway are most closely related to UC. 
Tab.3. Top 10 KEGG pathways of hub genes

\begin{tabular}{|lccl|}
\hline Term & Count & $\%$ & PValue \\
\hline Pathways in cancer & 16 & 61.54 & $3.36 \mathrm{E}-13$ \\
\hline Chagas disease & 14 & 53.85 & $4.42 \mathrm{E}-18$ \\
\hline Hepatitis B & 14 & 53.85 & $3.88 \mathrm{E}-16$ \\
\hline Toll-like receptor signaling pathway & 13 & 50.00 & $4.12 \mathrm{E}-16$ \\
\hline Influenza A & 13 & 50.00 & $1.84 \mathrm{E}-13$ \\
\hline Proteoglycans in cancer & 12 & 46.15 & $2.98 \mathrm{E}-11$ \\
\hline MAPK signaling pathway & 12 & 46.15 & $3.79 \mathrm{E}-10$ \\
\hline HTLV-I infection & 12 & 46.15 & $3.96 \mathrm{E}-10$ \\
\hline PI3K-Akt signaling pathway & 12 & 46.15 & $1.02 \mathrm{E}-08$ \\
\hline Prolactin signaling pathway & 11 & 42.31 & $2.05 \mathrm{E}-14$ \\
\hline
\end{tabular}

\section{Component-Target-Pathway Network}

The key targets of ZJP in treatment of UC enriched to 3 signaling pathways. We constructed components-targets-pathways network with 7 directly linked components screened by ADME, 18 directly regulated targets and 3 UC-related signaling pathways (Fig.9). The network showed that these 7 molecules were involved in regulating toll-like receptor signaling pathway, MAPK signaling pathway, and PI3K-Akt signaling pathway. In this network, we identify directed molecules and targets (Tab.4). In order to further characterize the safety of these active ingredients, toxicity prediction of 7 active components in ZJP were shown in tab.5. Six of seven active components ingredients in ZJP, namely, Obacunone, quercetin, Palmidin A, beta-sitosterol, isorhamnetin and rutaecarpine which corresponded to the hub genes were identified (Fig.10). From the Fig.10, we can see that quercetin, shared by Coptidis Rhizoma and Evodiae Fructus, targets most of the hub genes.

Tab.4. Directly linked components screened by OB and DL.

\begin{tabular}{|llllll|}
\hline $\begin{array}{l}\text { Code } \\
\text { name }\end{array}$ & $\begin{array}{l}\text { Molecule } \\
\text { Name }\end{array}$ & MW & $\begin{array}{l}\text { OB } \\
(\%)\end{array}$ & DL & Linked targets \\
\hline CR2 & Obacunone & 454.56 & 43.29 & 0.77 & MAPK1,MAPK14 \\
\hline CR3 & quercetin & 302.25 & 46.43 & 0.28 & AKT1,CXCL8,FOS,IL1B,IL2,IL6,JUN,MAPK1,MYC,PIK3CA,RELA,STAT1,TNF,TP53,VEGFA \\
\hline C9 & Palmidin A & 510.52 & 35.36 & 0.65 & MAPK1,PIK3CA \\
\hline C10 & Moupinamide & 313.38 & 86.71 & 0.26 & EGFR \\
\hline R48 & $\begin{array}{l}\text { beta- } \\
\text { sitosterol }\end{array}$ & 414.79 & 36.91 & 0.75 & JUN \\
\hline R78 & isorhamnetin & 316.28 & 49.6 & 0.31 & MAPK14,RELA \\
\hline R102 & rutaecarpine & 287.34 & 40.3 & 0.6 & TNF,IL4 \\
\hline
\end{tabular}

Tab.5. Toxicity prediction of 7 active components in ZJP. 


\begin{tabular}{|llllll|}
\hline Code name & Molecule Name & Ames mutagenesis & Hepatotoxicity & Acute Oral Toxicity & Acute oral toxicity evaluation \\
\hline CR2 & Obacunone & - & + & $51.827 \mathrm{mg} / \mathrm{kg}$ & Toxicity \\
\hline CR3 & quercetin & ++ & + & $698.794 \mathrm{mg} / \mathrm{kg}$ & low \\
\hline C9 & Palmidin A & + & - & $147.569 \mathrm{mg} / \mathrm{kg}$ & Toxicity \\
\hline C10 & Moupinamide & - & ++ & $1603.37 \mathrm{mg} / \mathrm{kg}$ & low \\
\hline R48 & beta-sitosterol & - & - & $273.371 \mathrm{mg} / \mathrm{kg}$ & Toxicity \\
\hline R78 & isorhamnetin & - & + & $604.02 \mathrm{mg} / \mathrm{kg}$ & low \\
\hline R102 & rutaecarpine & - & ++ & $624.265 \mathrm{mg} / \mathrm{kg}$ & low \\
\hline
\end{tabular}

Note: the "+" and "-" represent the predicted toxicity possibility. 0.1(-); 01-0.3(-); 0.3-05(-); 0.5-0.7(+); 0.7-0.9(++); 0.9-10(+++).Acute oral toxicity evaluation involves High-toxicity $(1 \sim 50 \mathrm{mg} / \mathrm{kg})$, Toxicity $(51 \sim 500 \mathrm{mg} / \mathrm{kg})$ and low-toxicity $(501 \sim 5000 \mathrm{mg} / \mathrm{kg})$.

\section{Discussion}

The pathogenesis of ulcerative colitis is sophisticated, including genetic, environmental, psychological stress and other factors ${ }^{[10]}$. In view of the complicated pathogenesis of ulcerative colitis, a single target drug hardly obtain better therapeutic effect, and traditional Chinese medicine (TCM) has multi-target effect, which has become a considerable source of UC therapeutic drugs ${ }^{[11-12]}$. Network pharmacology provides a new strategy for the research of TCM through exploring the relationship between drugs and diseases from a holistic perspective. ZJP is composed of Coptis chinensis and Evodia rutaecarpa, which has the material basis for the treatment of UC. For example, berberine inhibited p38 MAPK

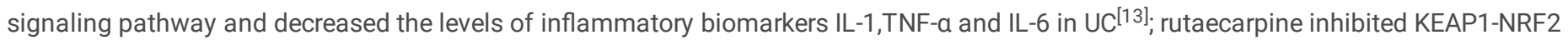
interaction to activate NRF2 and ameliorate dextran sulfate sodium-induced colitis ${ }^{[14]}$.However, as a traditional Chinese medicine compound, the mechanism of ZJP on UC is not clear, and other effective components are still worth exploring. In this study, we successfully identified 7 active molecules and 3 important pathways related to UC through network pharmacology and bioinformatics. This method can more clearly identify the targets and specific mechanisms of TCM in the treatment of diseases and obtain the key active molecules, which is of great significance for the development of natural drugs and the treatment of diseases.

\section{Analysis of potential targets}

In the ingredient-target-disease interactive network, The highest degree target was PTGS2. The following targets were PTGS1, ADRB2, NOS3区 CALM1. PTGS2 can encode COX-2, which is related to inflammation and oxidative stress. Studies have shown that COX-2-PGE 2 signal transduction affects the inflammation level and intestinal epithelial regeneration of UC ${ }^{[15]}$. On the contrary, COX-1 protein regulated by PTGS1 gene is a considerable protective mediator of UC ${ }^{[16]}$. ADRB2 gene deletion is more sensitive to intestinal inflammation. $\beta 2$ adrenergic receptor regulated by ADRB2 gene controls UC inflammation by driving rapid IL-10 secretion ${ }^{[17]}$. NOS3 regulates eNOS and plays a protective role in trinitrobenzene sulfonic acid-induced colitis by regulating inflammation ${ }^{[18]}$. CALM1 gene is associated with inflammation and immune regulation ${ }^{[19-20]}$. In summary, ZJP is closely related to the regulation of PTGS2, PTGS1, ADRB2, NOS3, and CALM1 in the treatment of UC. ZJP is very likely to regulate the inflammation and immune response of UC through these target genes. Above all, ZJP treatment of UC may regulate inflammation and immune response through PTGS2, PTGS1, ADRB2, NOS3 and CALM1 target genes.

\section{Analysis of PPI network}

The target of JUN, MAPK1, TNF, PIK3CA and RELA were overexpressed in PPI network. This result reveals the pivotal molecular targets involved in the regulation of ZJP in the treatment of UC. JUN participates in cell proliferation and apoptosis. Inhibition of c-jun protein regulated by JUN can alleviate oxidative stress and promote mucosal healing [21]; Studies have shown that inhibition of MAPK1 expression can inhibit cell inflammation and apoptosis, thereby relieving UC [22]; TNF plays an indispensable role in the pathogenesis of inflammatory bowel disease. Randomized controlled trials have confirmed that anti TNF - a biotherapy is effective for UC [23]. PIK3CA gene can regulate PI3K/Akt pathway, which is closely related to UC. A large number of studies have shown that inhibiting PI3K/Akt can reduce the inflammation and oxidative stress induced by DSS in UC ${ }^{[24-25]}$. RELA is involved in the response of cells to external stimuli. Research has found that inhibition of RELA can alleviate the inflammation and oxidative stress of UC [26].

\section{Analysis of signaling pathways}

GO-BP enrichment analysis showed that ZJP may play an anti-UC role by regulating inflammatory response, immune response, apoptosis, MAPK cascade, signal transduction and other biological functions. From two aspects of network construction and central network evaluation, 
26 key targets of ZJP potential target network were selected which were significantly enriched in three UC related signaling pathways, including PI3K-Akt signaling pathway, MAPK signaling pathway and toll like receptor signaling pathway. Studies have shown that PI3K-Akt signaling pathway can regulate the inflammation and oxidative stress of UC [27-28]; MAPK pathway plays a key role in regulating the development of UC inflammation [29]; Toll-like receptor signaling pathway plays an important role in the immune system and participates in the inflammatory process of UC ${ }^{[30]}$. However, the key target of this study enriched in toll like receptor signaling pathway is still focused on the downstream MAPK pathway crosstalk, and it can be considered that ZJP treatment of UC mainly directly regulates PI3K-Akt signaling pathway and MAPK signaling pathway. Fig.11 summarize the representative pathways in the progress of UC and the key targets (red nodes) related to ZJP, indicating that ZJP treatment of UC may be closely related to the regulation of inflammation and immunity.

\section{Analysis of active ingredients}

In the ingredient-target-disease interactive network, key active ingredients with higher degree includes quercetin, isorhamnetin, caffeine, (R)Canadine, magnoflorine. Among them, quercetin and isorhamnetin were identified by the Component-Target-Pathway network as directly involved in the pathway regulation of UC, and their toxicity is predicted to be low. In other words, quercetin and isorhamnetin may be safe and

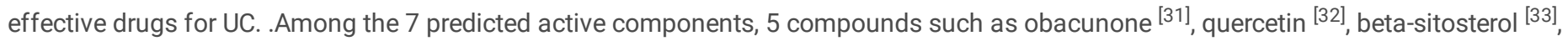
isorhamnetin ${ }^{[34]}$ and rutaecarpine ${ }^{[35]}$ have been proved to be effective in the treatment of UC. Palmidin A and moupinamide have not been reported in relevant studies, which is worthy of further exploration. The above evidences preliminarily suggest that these components of ZJP obtained by network pharmacology analysis may be effective components in the treatment of UC, but further experimental verification and mechanism research are needed.

In this study, the key target, effective components and key signal pathway of ZJP in UC treatment were systematically revealed in the network pharmacology, which provided the direction and basis for further research in the future. However, there are some limitations in using network pharmacology to predict active ingredients and potential mechanisms. Firstly, it is difficult to distinguish the inhibition effect and activation effect of potential targets. Secondly, the prediction results may be affected by the deviation of the path/function with high research frequency. Therefore, it is necessary and valuable to verify the potential active ingredients by experiments to verify the theoretical prediction, and the network pharmacology method is still valuable as a useful screening reference tool.

\section{Conclusion}

ZJP on UC by use of network pharmacological approach, which reflects the multi-component, multi-target and integrated regulation of TCM prescriptions, and provides a basis for further study of the pharmacological component and mechanism of ZJP in treatment of UC. Immunity regulation and inflammatory regulation are the crucial regulation cores in this mechanism.

\section{Abbreviations}

ZJP, Zuojin Pill; UC, ulcerative colitis; TCMSP

Traditional Chinese Medicine Systems Pharmacology; DL, Drug-likeness; OB, Oral bioavailability; DAVID, Annotation, Visualization and Integrated Discovery; KEGG, Kyoto Encyclopedia of Genes and Genomes; GO, gene ontology; BP, biological process; STRING, Search Tool for the Retrieval of Interacting Genes/Proteins; PPI, protein-protein interactions; PTGS2, prostaglandinendoperoxide Synthase 2; PTGS1, prostaglandinendoperoxide Synthase 1; ADRB2, $\beta 2$ adrenergic receptor; NOS3, nitric oxide synthase 3; CALM1, Calmodulin-1; MAPK, mitogenactivated protein kinase; PI3K, phosphatidylinositol-3-kinase; Akt, protein kinase $\mathrm{B}$.

\section{Declarations}

\section{Acknowledgements}

The authors would like to thank the authors of all references.

\section{Authors' contributions}

Ying Wei conducted the analytical part, wrote the first version of the manuscript, and downloaded the reference and processed the graph and the table in the manuscript. Sichen Ren and Ruilin Wang finalized the manuscript. Manyi Jing, Honghong Liu and Min Wang collected the data. Hongtao Song (corresponding author) and Yanling Zhao (corresponding author) conceived and coordinated the study, and critically evaluated the data.

\section{Funding}

This study was financially supported by the National Key Research and Development Program (No. 2018YFC1704500). 
All data are available in the manuscript and they are showed in figures and tables.

\section{References}

1. Ordás I, Eckmann L, Talamini M, Baumgart DC, Sandborn WJ. Ulcerative colitis. Lancet. 2012 Nov 3;380(9853):1606-19. doi: 10.1016/S0140-6736(12)60150-0. Epub 2012 Aug 20. PMID: 22914296.

2. Wei SC, Sollano J, Hui YT, Yu W, Santos Estrella PV, Llamado LJQ, Koram N. Epidemiology, burden of disease, and unmet needs in the treatment of ulcerative colitis in Asia. Expert Rev Gastroenterol Hepatol. 2021 Jan 19:1-15. doi: 10.1080/17474124.2021.1840976. Epub ahead of print. PMID: 33107344.

3. Sehgal P, Colombel JF, Aboubakr A, Narula N. Systematic review: safety of mesalazine in ulcerative colitis. Aliment Pharmacol Ther. 2018 Jun;47(12):1597-1609. doi: 10.1111/apt.14688. Epub 2018 May 3. PMID: 29722441.

4. Koning M, Ailabouni R, Gearry RB, Frampton CM, Barclay ML. Use and predictors of oral complementary and alternative medicine by patients with inflammatory bowel disease: a population-based, case-control study. Inflamm Bowel Dis. 2013 Mar-Apr;19(4):767 - 78. doi: 10.1097/MIB.0b013e31827f27c8. PMID: 23429459.

5. Weizman AV, Ahn E, Thanabalan R, Leung W, Croitoru K, Silverberg MS, Steinhart AH, Nguyen GC. Characterisation of complementary and alternative medicine use and its impact on medication adherence in inflammatory bowel disease. Aliment Pharmacol Ther. 2012 Feb;35(3):342-9. doi:10.1111/j.1365-2036.2011.04956.x. Epub 2011 Dec 18. PMID: 22176478.

6. Zhou BG, Liu FC, Zhao HM, Zhang XY, Wang HY, Liu DY. Regulatory effect of Zuojin Pill on correlation with gut microbiota and Treg cells in DSS-induced colitis. J Ethnopharmacol. 2020 Nov 15;262:113211. doi: 10.1016/j.jep.2020.113211. Epub 2020 Jul 30. PMID: 32739566.

7. Xiang SY, Zhao J, Lu Y, Chen RM, Wang Y, Chen Y, Long B, Zhu LP, Yao PF, Xu YF, Chen JH. Network pharmacology-based identification for therapeutic mechanism of Ling-Gui-Zhu-Gan decoction in the metabolic syndrome induced by antipsychotic drugs. Comput Biol Med. 2019 Jul;110:1-7. doi: 10.1016/j.compbiomed.2019.05.007. Epub 2019 May 7. PMID: 31085379.

8. Zhu B, Zhang W, Lu Y, Hu S, Gao R, Sun Z, Chen X, Ma J, Guo S, Du S, Li P. Network pharmacology-based identification of protective mechanism of Panax Notoginseng Saponins on aspirin induced gastrointestinal injury. Biomed Pharmacother. 2018 Sep;105:159-166. doi: 10.1016/j.biopha.2018.04.054. Epub 2018 May 29. PMID: 29857294.

9. Li AP, He SS, Zhang WN, Zhang LC, Liu YT, Li K, Qin XM. Exploration the active compounds of Astragali Radix in treatment of adriamycin nephropathy by network pharmacology combined with transcriptomic approach. J Ethnopharmacol. 2020 Aug 10;258:112537. doi: 10.1016/j.jep.2019.112537. Epub 2020 Jan 1. PMID: 31901455.

10. Ordás I, Eckmann L, Talamini M, Baumgart DC, Sandborn WJ. Ulcerative colitis. Lancet. 2012 Nov 3;380(9853):1606-19. doi: 10.1016/S0140-6736(12)60150-0. Epub 2012 Aug 20. PMID: 22914296.

11. Ye B, Shen H, Lu Y, Wang YQ. Clinical observations on 100 cases of ulcerative colitis treated with the method of clearing away heat, expelling dampness, promoting blood circulation and healing ulcer. J Tradit Chin Med. 2010 Jun;30(2):98-102. doi: 10.1016/s02546272(10)60022-2. PMID: 20653164.

12. Chen XL, Wen Y, Wu ZC, Zhang BP, Hou ZK, Xiao JL, Lin MQ, Hu Y, Wu ZL, Deng JM, Liu FB, Liu TW. Development of a Traditional Chinese Medicine Syndrome-Specific Scale for Ulcerative Colitis: The Large Intestine Dampness-Heat Syndrome Questionnaire. Evid Based Complement Alternat Med. 2018 Jul 12;2018:4039019. doi: 10.1155/2018/4039019. PMID: 30108653; PMCID: PMC6077564.

13. Jia L, Xue K, Liu J, Habotta OA, Hu L, Abdel Moneim AE. Anticolitic Effect of Berberine in Rat Experimental Model: Impact of PGE2/p38 MAPK Pathways. Mediators Inflamm. 2020 Sep 29;2020:9419085. doi: 10.1155/2020/9419085. PMID: 33061833.

14. Zhang Y, Yan T, Sun D, Xie C, Wang T, Liu X, Wang J, Wang Q, Luo Y, Wang P, Yagai T, Krausz KW, Yang X, Gonzalez FJ. Rutaecarpine inhibits KEAP1-NRF2 interaction to activate NRF2 and ameliorate dextran sulfate sodium-induced colitis. Free Radic Biol Med. 2020 Feb 20;148:33-41. doi: 10.1016/j.freeradbiomed.2019.12.012. Epub 2019 Dec 23. PMID: 31874248; PMCID: PMC7376370.

15. Li Y, Soendergaard C, Bergenheim FH, Aronoff DM, Milne G, Riis LB, Seidelin JB, Jensen KB, Nielsen OH. COX-2-PGE2 Signaling Impairs Intestinal Epithelial Regeneration and Associates with TNF Inhibitor Responsiveness in Ulcerative Colitis. EBioMedicine. 2018 Oct;36:497507. doi: 10.1016/j.ebiom.2018.08.040. Epub 2018 Sep 3. PMID: 30190207; PMCID: PMC6197735.

16. Peng X, Li J, Tan S, Xu M, Tao J, Jiang J, Liu H, Wu B. COX-1/PGE2/EP4 alleviates mucosal injury by upregulating $\beta$-arr1-mediated Akt signaling in colitis. Sci Rep. 2017 Apr 21;7(1):1055. doi: 10.1038/s41598-017-01169-6. PMID: 28432343; PMCID: PMC5430694.

17. Ağaç D, Estrada LD, Maples R, Hooper LV, Farrar JD. The $\beta 2$-adrenergic receptor controls inflammation by driving rapid IL-10 secretion. Brain Behav Immun. 2018 Nov;74:176-85. doi:10.1016/j.bbi.2018.09.004. Epub 2018 Sep 5. PMID: 30195028; PMCID: PMC6289674.

18. Vallance BA, Dijkstra G, Qiu B, van der Waaij LA, van Goor H, Jansen PL, Mashimo H, Collins SM. Relative contributions of NOS isoforms during experimental colitis: endothelial-derived NOS maintains mucosal integrity. Am J Physiol Gastrointest Liver Physiol. 2004 
Oct;287(4):G865-74. doi:10.1152/ajpgi.00187.2004. Epub 2004 Jun 24. PMID: 15217783.

19. Bueno-Silva B, Rosalen PL, Alencar SM, Mayer MPA. Vestitol drives LPS-activated macrophages into M2 phenotype through modulation of NF-KB pathway. Int Immunopharmacol. 2020 Feb 27;82:106329. doi: 10.1016/j.intimp.2020.106329. Epub ahead of print. PMID:

32114412.

20. Kim J, Bissonnette R, Lee J, Correa da Rosa J, Suárez-Fariñas M, Lowes MA, Krueger JG. The Spectrum of Mild to Severe Psoriasis Vulgaris Is Defined by a Common Activation of IL-17 Pathway Genes, but with Key Differences in Immune Regulatory Genes. J Invest Dermatol. 2016 Nov;136(11):2173-2182. doi: 10.1016/j.jid.2016.04.032. Epub 2016 May 13. PMID: 27185339.

21. Sharma A, Tirpude NV, Kulurkar PM, Sharma R, Padwad Y. Berberis lycium fruit extract attenuates oxi-inflammatory stress and promotes mucosal healing by mitigating NF-KB/c-Jun/MAPKs signalling and augmenting splenic Treg proliferation in a murine model of dextran sulphate sodium-induced ulcerative colitis. Eur J Nutr. 2020 Sep;59(6):2663-81. doi:10.1007/s00394-019-02114-1. Epub 2019 Oct 16. PMID: 31620885

22. Gao W, Wang C, Yu L, Sheng T, Wu Z, Wang X, Zhang D, Lin Y, Gong Y. Chlorogenic Acid Attenuates Dextran Sodium Sulfate-Induced Ulcerative Colitis in Mice through MAPK/ERK/JNK Pathway. Biomed Res Int. 2019 Apr 18;2019:6769789. doi: 10.1155/2019/6769789. PMID: 31139644; PMCID: PMC6500688.

23. Sands BE, Kaplan GG. The role of TNFalpha in ulcerative colitis. J Clin Pharmacol. 2007 Aug;47(8):930 - 41. doi: 10.1177/0091270007301623. Epub 2007 Jun 13. PMID: 17567930.

24. Peng KY, Gu JF, Su SL, Zhu Y, Guo JM, Qian DW, Duan JA. Salvia miltiorrhiza stems and leaves total phenolic acids combination with tanshinone protect against DSS-induced ulcerative colitis through inhibiting TLR4/PI3K/AKT/mTOR signaling pathway in mice. J Ethnopharmacol. 2021 Jan 10;264:113052. doi: 10.1016/j.jep.2020.113052. Epub 2020 Jun 11. PMID: 32535239.

25. Liu B, Piao X, Niu W, Zhang Q, Ma C, Wu T, Gu Q, Cui T, Li S. Kuijieyuan Decoction Improved Intestinal Barrier Injury of Ulcerative Colitis by Affecting TLR4-Dependent PI3K/AKT/NF-KB Oxidative and Inflammatory Signaling and Gut Microbiota. Front Pharmacol. 2020 Jul 29;11:1036. doi: 10.3389/fphar.2020.01036. PMID: 32848725; PMCID: PMC7403404.

26. Shen J, Cheng J, Zhu S, Zhao J, Ye Q, Xu Y, Dong H, Zheng X. Regulating effect of baicalin on IKK/IKB/NF-kB signaling pathway and apoptosis-related proteins in rats with ulcerative colitis. Int Immunopharmacol. 2019 Aug;73:193-200. doi: 10.1016/j.intimp.2019.04.052. Epub 2019 May 16. PMID: 31103874.

27. Li MY, Luo HJ, Wu X, Liu YH, Gan YX, Xu N, Zhang YM, Zhang SH, Zhou CL, Su ZR, Huang XQ, Zheng XB. Anti-Inflammatory Effects of Huangqin Decoction on Dextran Sulfate Sodium-Induced Ulcerative Colitis in Mice Through Regulation of the Gut Microbiota and Suppression of the Ras-PI3K-Akt-HIF-1a and NF-KB Pathways. Front Pharmacol. 2020 Jan 20;10:1552. doi: 10.3389/fphar.2019.01552. PMID: 32038240; PMCID: PMC6984456.

28. Liu B, Piao X, Niu W, Zhang Q, Ma C, Wu T, Gu Q, Cui T, Li S. Kuijieyuan Decoction Improved Intestinal Barrier Injury of Ulcerative Colitis by Affecting TLR4-Dependent PI3K/AKT/NF-KB Oxidative and Inflammatory Signaling and Gut Microbiota. Front Pharmacol. 2020 Jul 29;11:1036. doi: 10.3389/fphar.2020.01036. PMID: 32848725; PMCID: PMC7403404.

29. Zobeiri M, Momtaz S, Parvizi F, Tewari D, Farzaei MH, Nabavi SM. Targeting Mitogen-Activated Protein Kinases by Natural Products: A Novel Therapeutic Approach for Inflammatory Bowel Diseases. Curr Pharm Biotechnol. 2020;21(13):1342-1353. doi: 10.2174/1389201021666191216122555. PMID: 31840607.

30. Kordjazy N, Haj-Mirzaian A, Haj-Mirzaian A, Rohani MM, Gelfand EW, Rezaei N, Abdolghaffari AH. Role of toll-like receptors in inflammatory bowel disease. Pharmacol Res. 2018 Mar;129:204-215. doi: 10.1016/j.phrs.2017.11.017. Epub 2017 Nov 16. PMID: 29155256.

31. Luo X, Yue B, Yu Z, Ren Y, Zhang J, Ren J, Wang Z, Dou W. Obacunone Protects Against Ulcerative Colitis in Mice by Modulating Gut Microbiota, Attenuating TLR4/NF-KB Signaling Cascades, and Improving Disrupted Epithelial Barriers. Front Microbiol. 2020 Mar 31;11:497. doi: 10.3389/fmicb.2020.00497. PMID: 32296403; PMCID: PMC7136403.

32. Habtemariam S, Belai A. Natural Therapies of the Inflammatory Bowel Disease: The Case of Rutin and its Aglycone, Quercetin. Mini Rev Med Chem. 2018;18(3):234-243. doi: 10.2174/1389557517666170120152417. PMID: 28117024.

33. Ding K, Tan YY, Ding Y, Fang Y, Yang X, Fang J, Xu DC, Zhang H, Lu W, Li M, Huang SC, Cai ML, Song Y, Ding YJ, Zhang SM. $\beta$-Sitosterol improves experimental colitis in mice with a target against pathogenic bacteria. J Cell Biochem. 2019 Apr;120(4):5687-5694. doi: 10.1002/jcb.27853. Epub 2018 Dec 11. PMID: 30548286.

34. Dou W, Zhang J, Li H, Kortagere S, Sun K, Ding L, Ren G, Wang Z, Mani S. Plant flavonol isorhamnetin attenuates chemically induced inflammatory bowel disease via a PXR-dependent pathway. J Nutr Biochem. 2014 Sep;25(9):923-33. doi:10.1016/j.jnutbio.2014.04.006. Epub 2014 May 9. PMID: 24913217; PMCID: PMC4125479.

35. Luo DN, Li FJ, Zou YY. [Therapeutic effects of rutaecarpine on dextran sodium sulfate-induced experimental colitis in mice]. Zhonghua Yi Xue Za Zhi. 2018 Feb 13;98(7):533-538. Chinese. doi: 10.3760/cma.j.issn.0376-2491.2018.07.010. PMID: 29495224. 
Figures

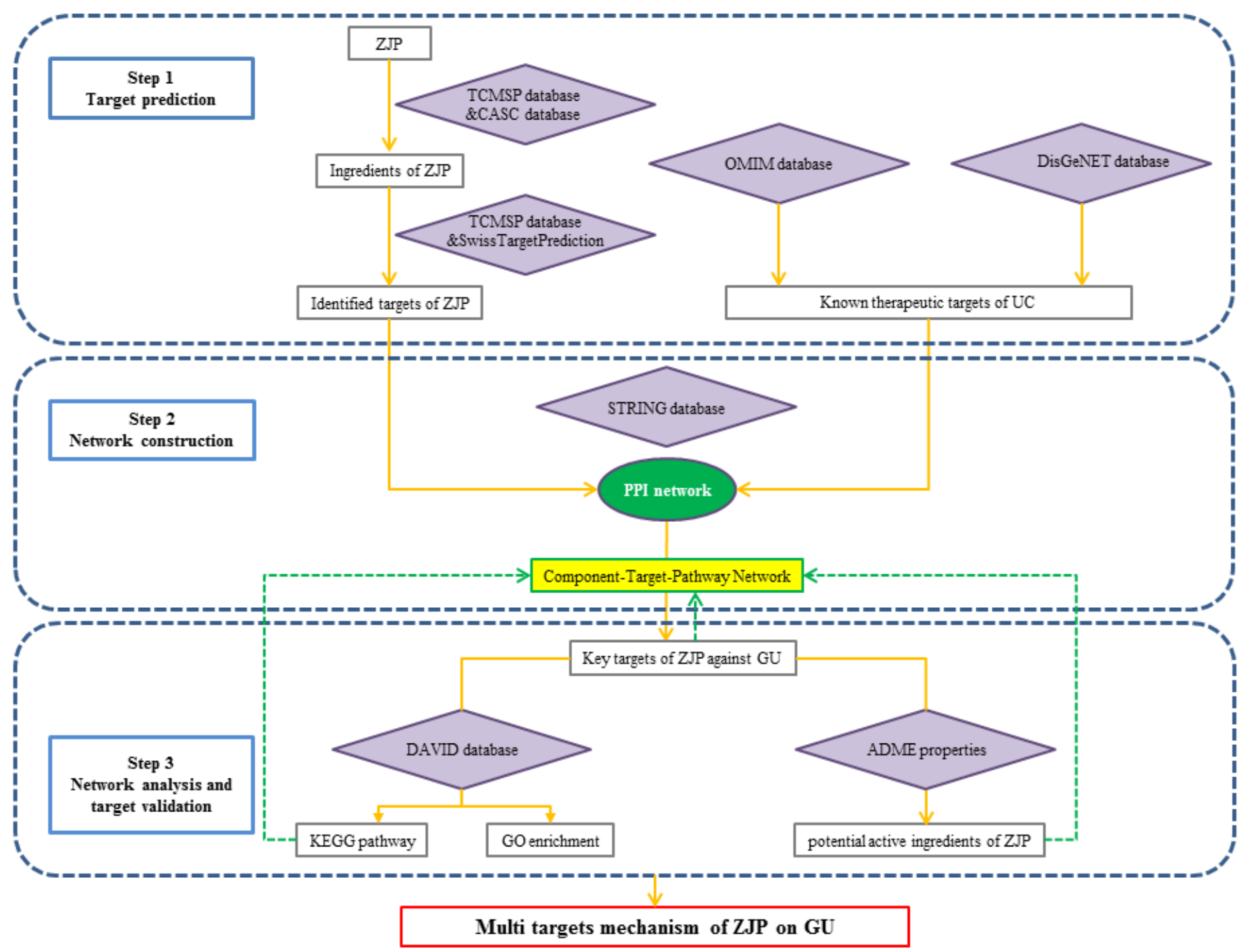

Figure 1

The flowchart of network pharmacology-based strategy for deciphering the mechanisms of ZJP acting on UC. 
A

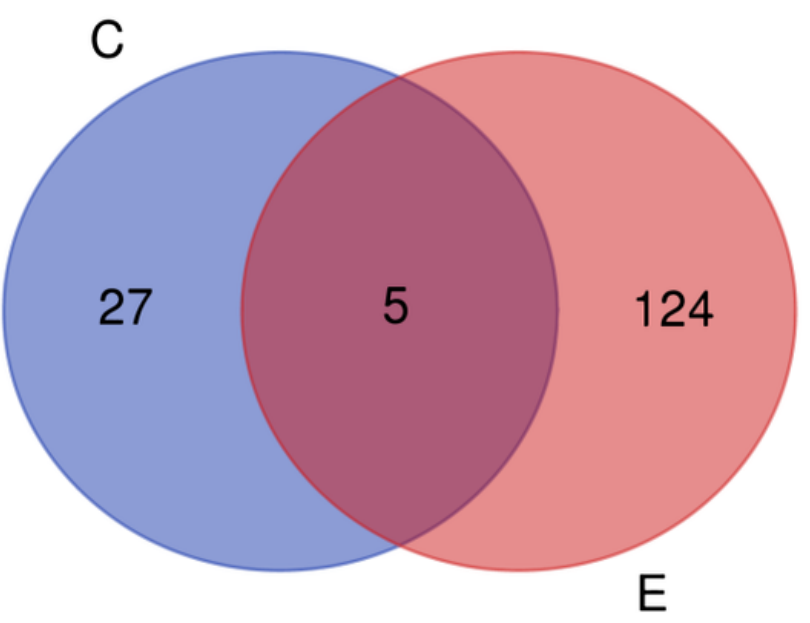

C

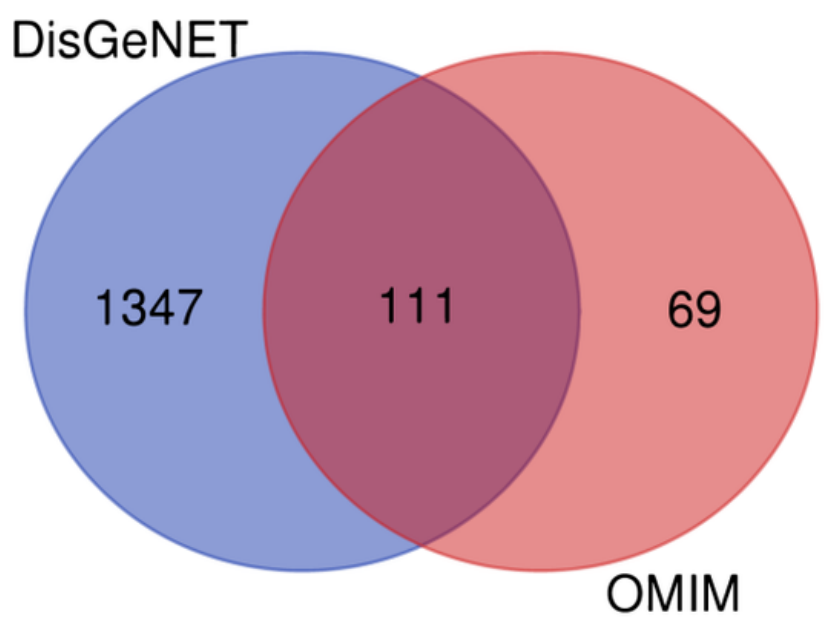

B

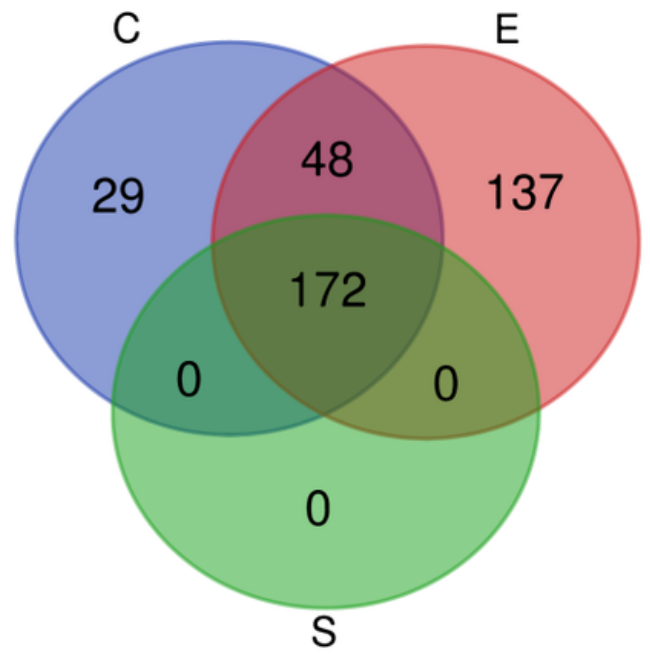

D

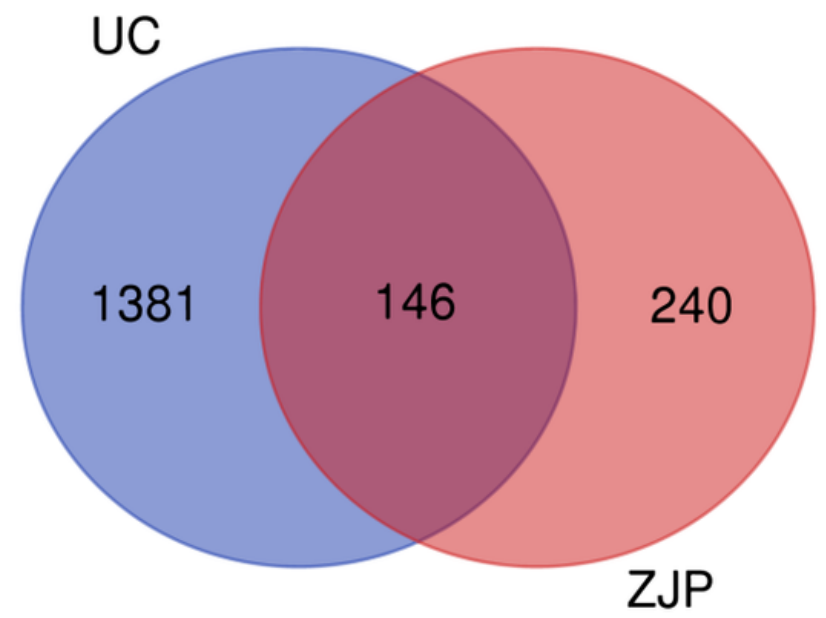

Figure 2

Distribution of Ingredients and targets. 


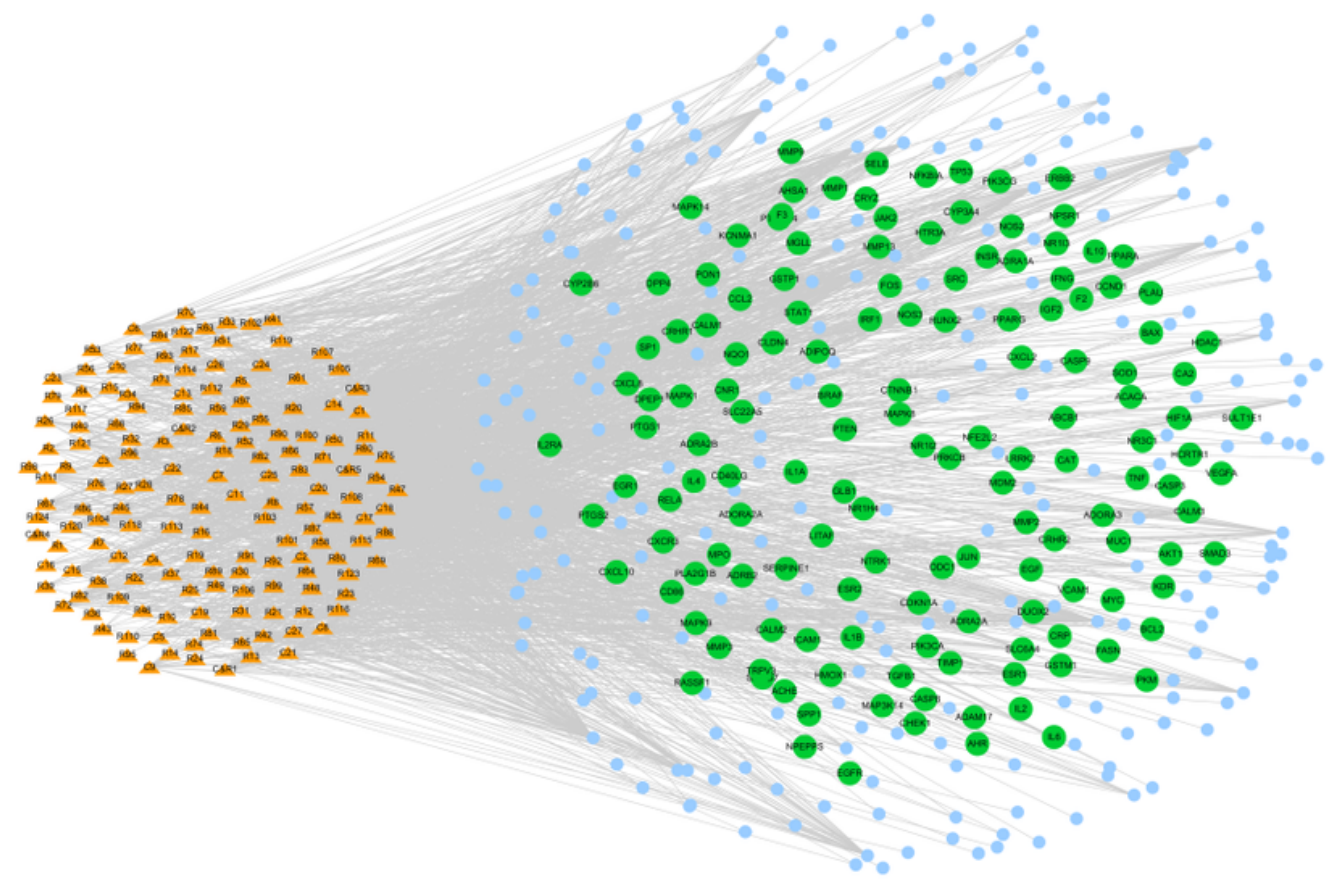

Figure 3

The ingredient-target-disease interactive network (The orange triangle nodes represent ingredients in ZJP, the blue round nodes represent the targets of drugs and the disease, and the green round nodes represent the common targets of drugs and disease)

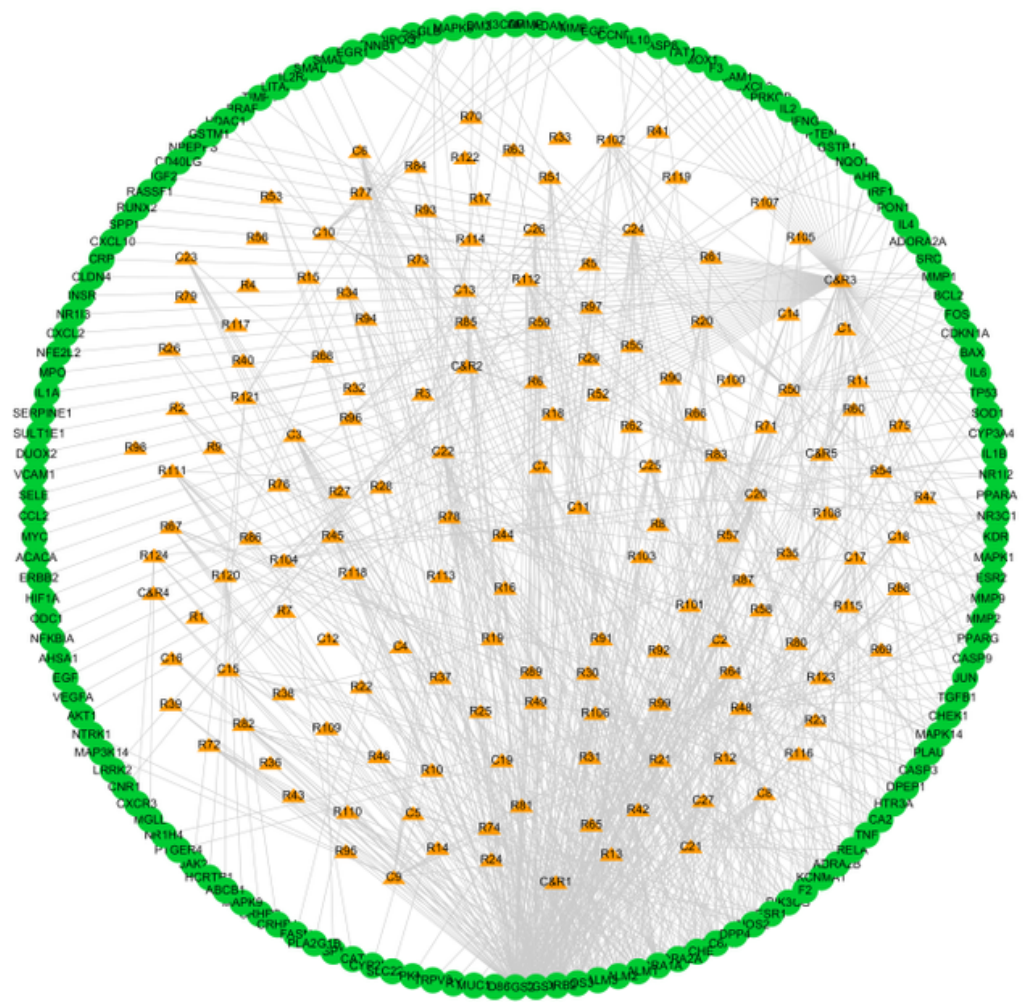

Figure 4 
The network of ingredients and common targets of drugs and the disease (The orange triangle nodes represent possible active ingredients in Xiaochaihu decoction and the green round nodes represent the common targets of drugs and disease)

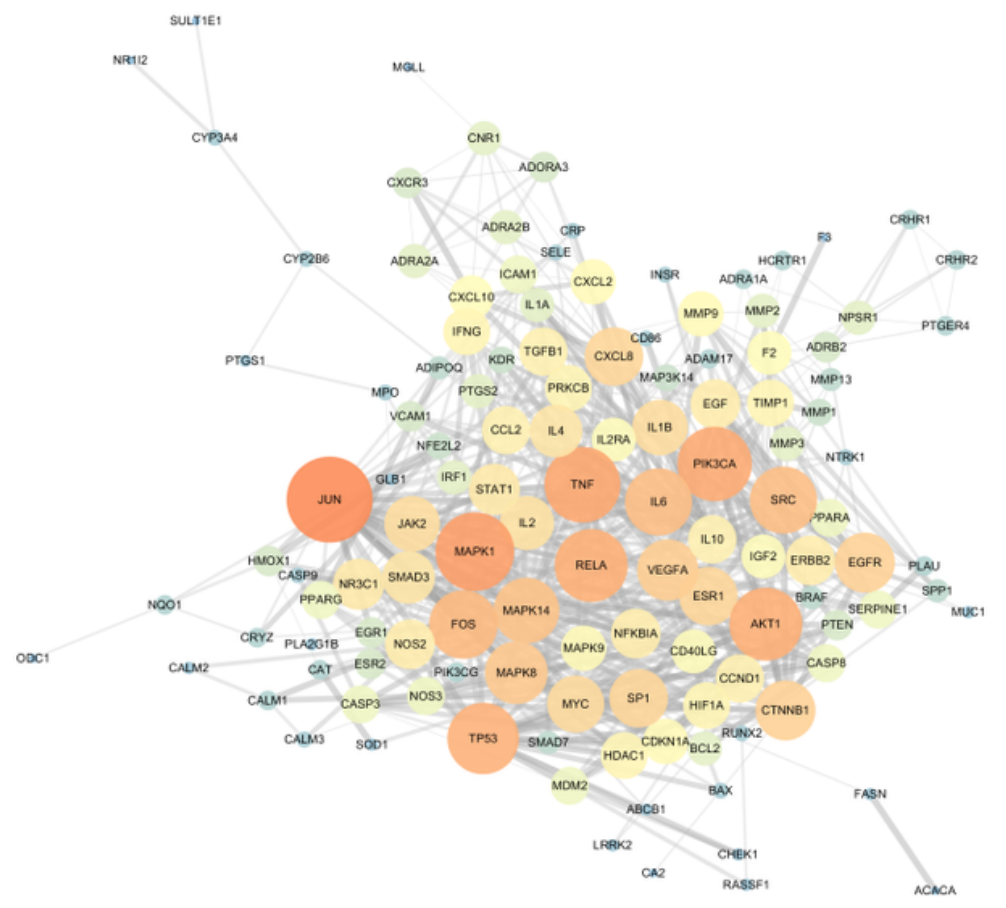

\section{Figure 5}

The PPI network of 146 common targets. The higher the degree value is, the brighter the color is and the larger the node is.

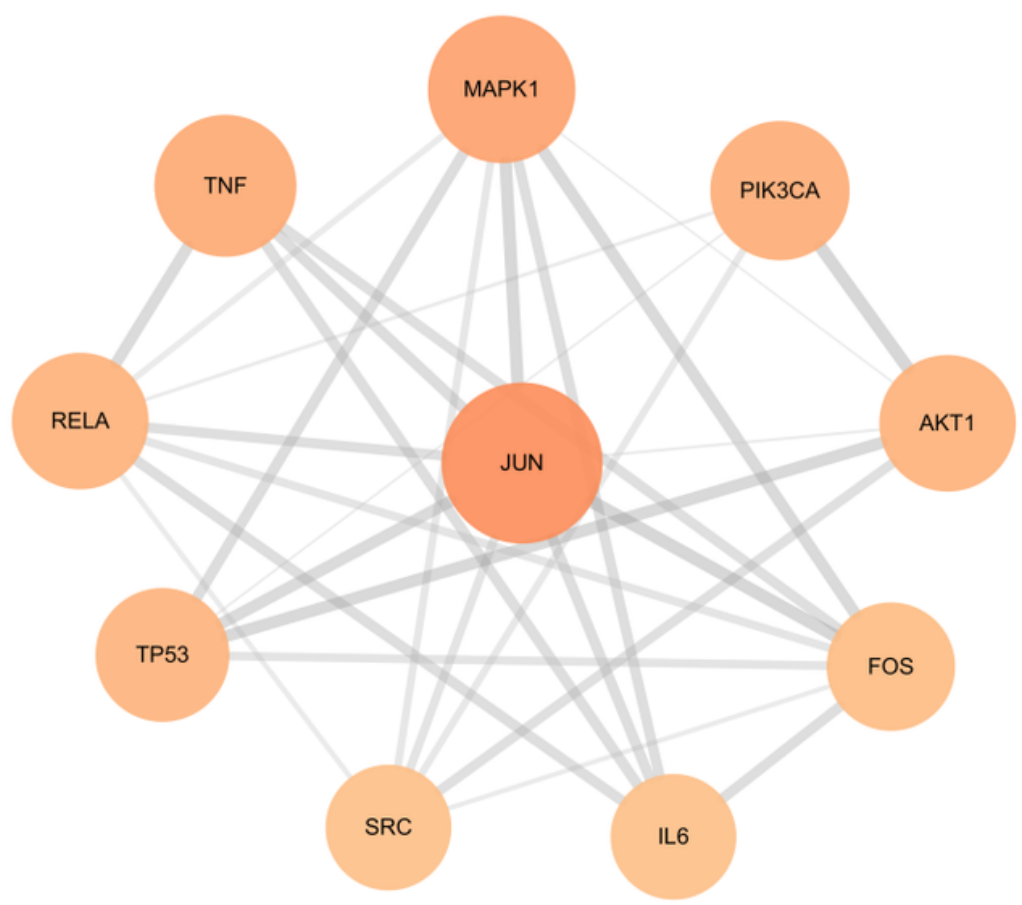

Figure 6 
The interaction network of top 10 identified targets. The higher the degree value is, the brighter the color is and the larger the node is.

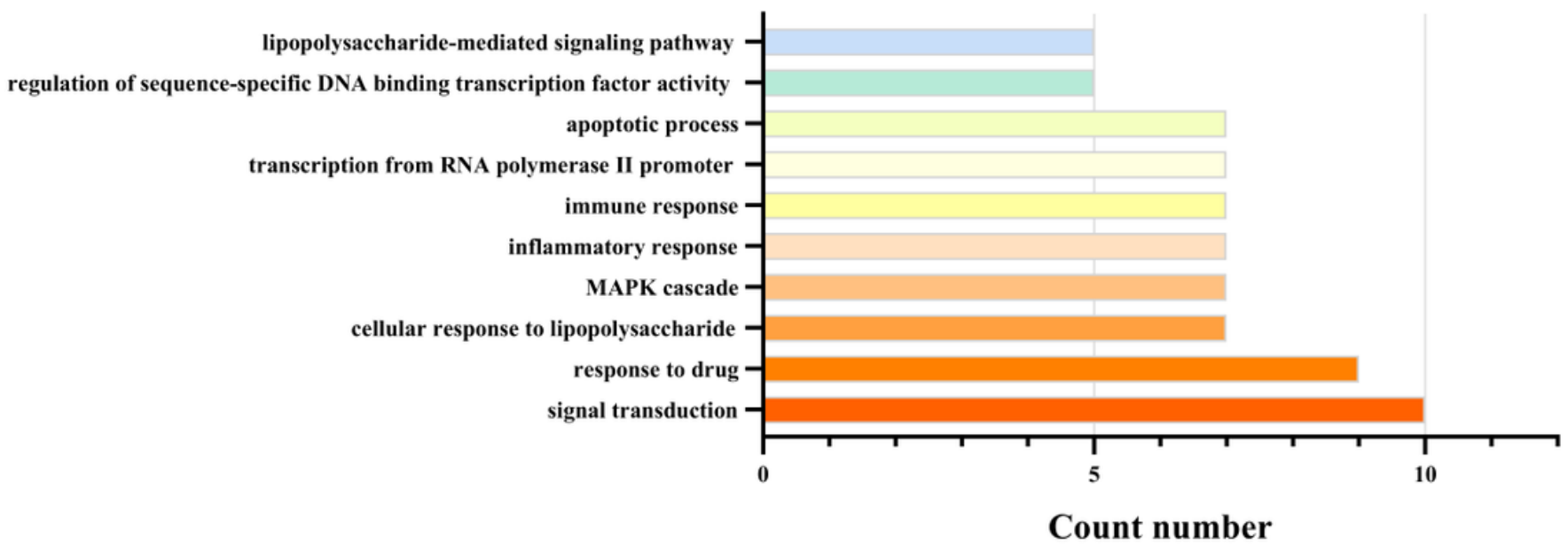

Figure 7

Top $10 \mathrm{GO}$ terms of hub genes.

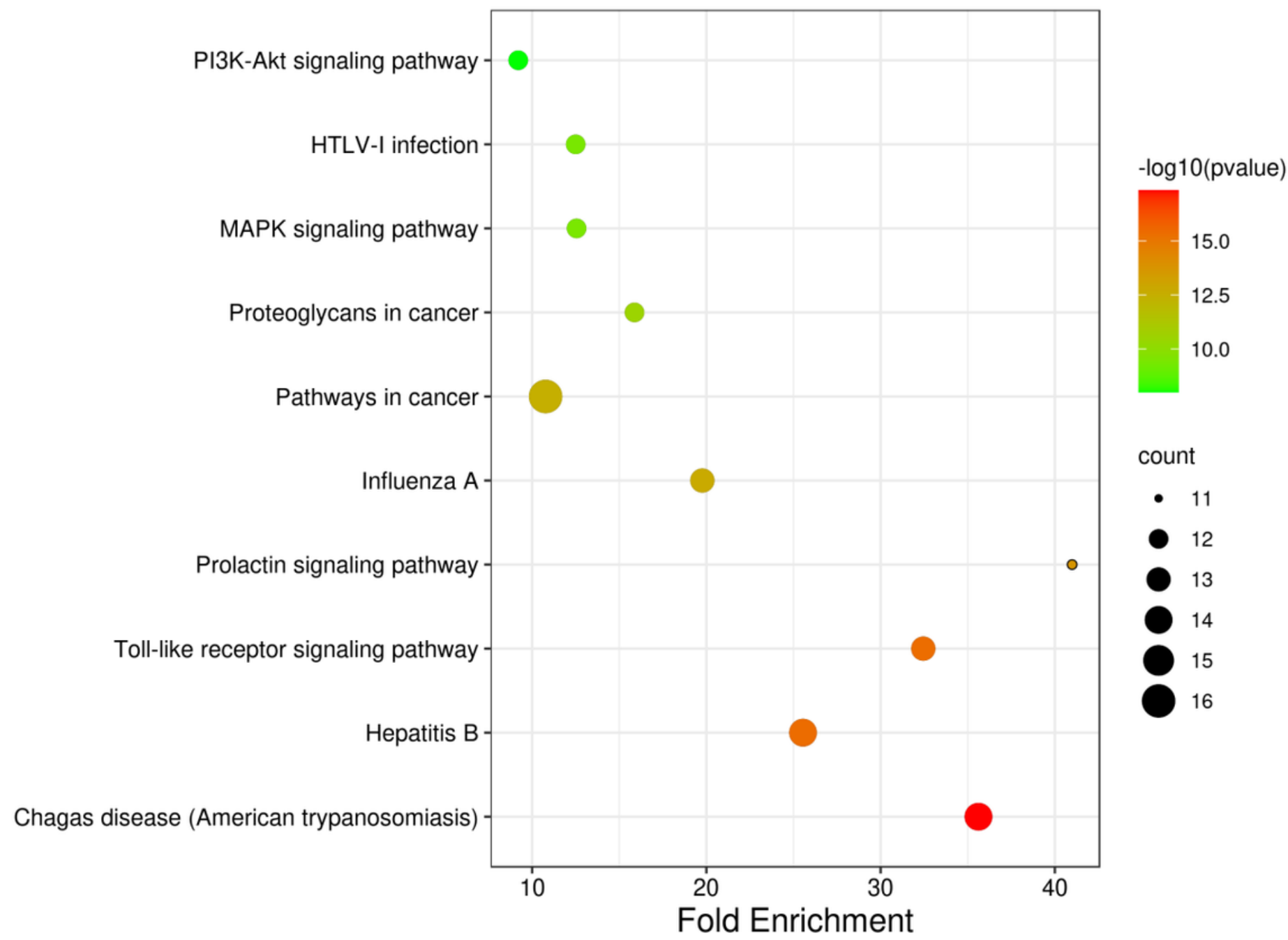

Figure 8 
Top 10 KEGG terms of hub genes.

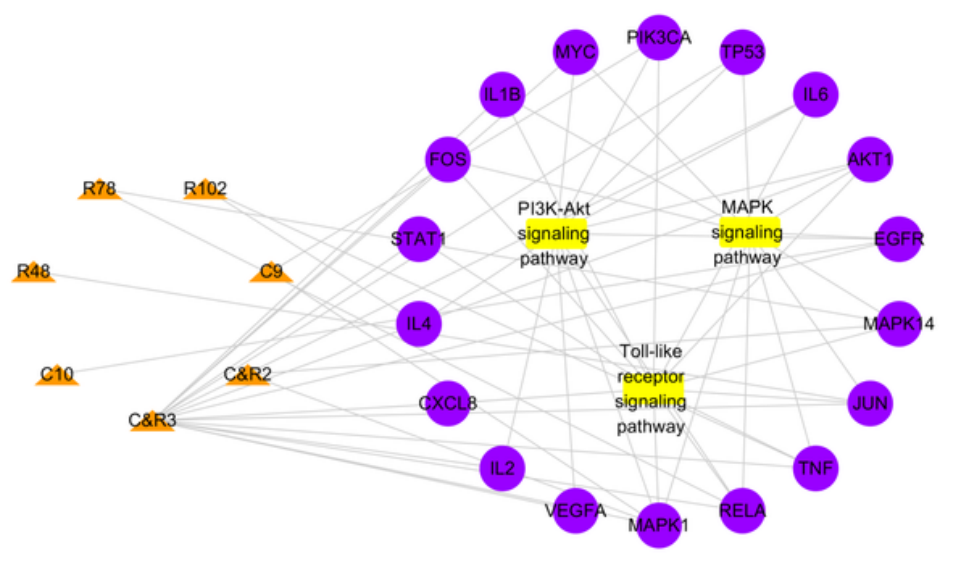

\section{Figure 9}

Component-Target-Pathway Network.
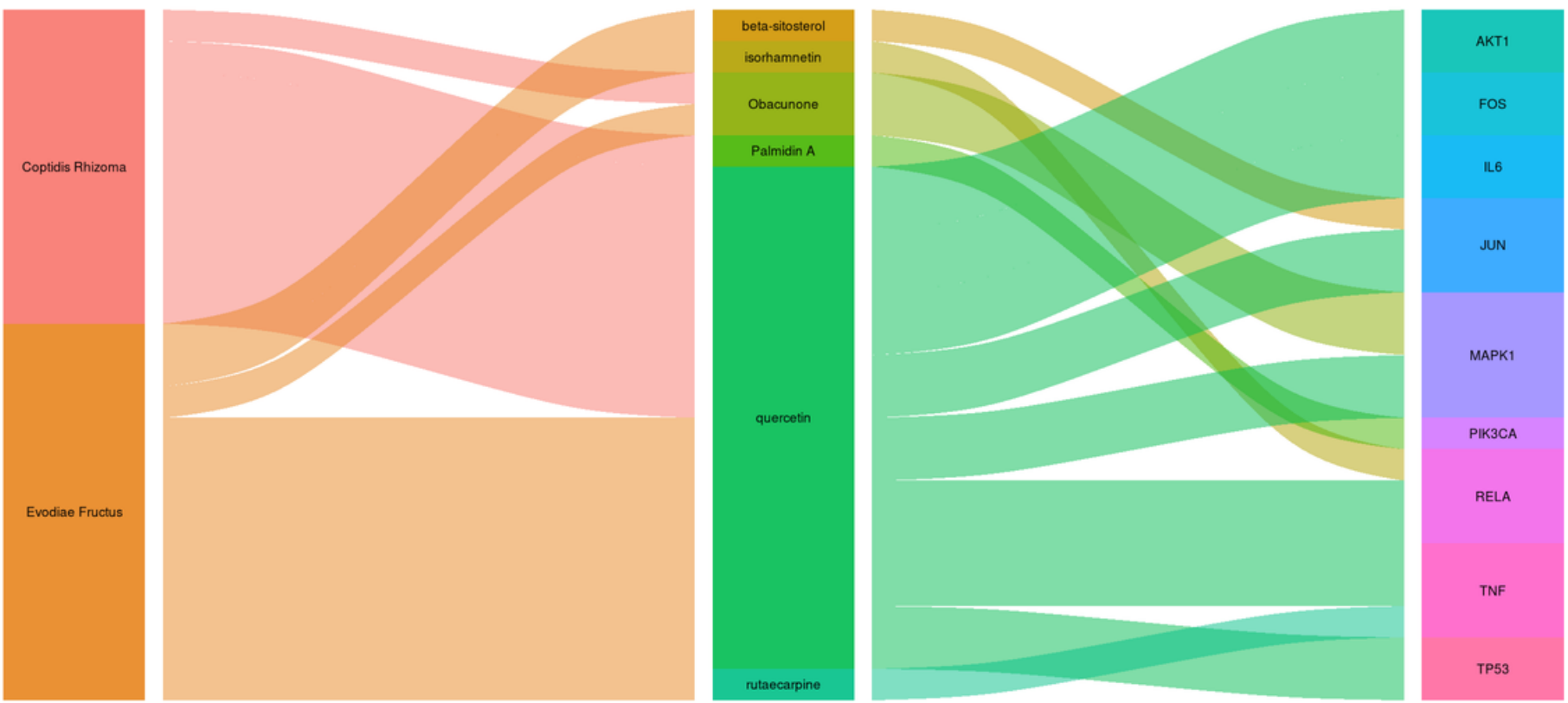

\section{Figure 10}

The relationship between herbs, ingredients, and targets. 


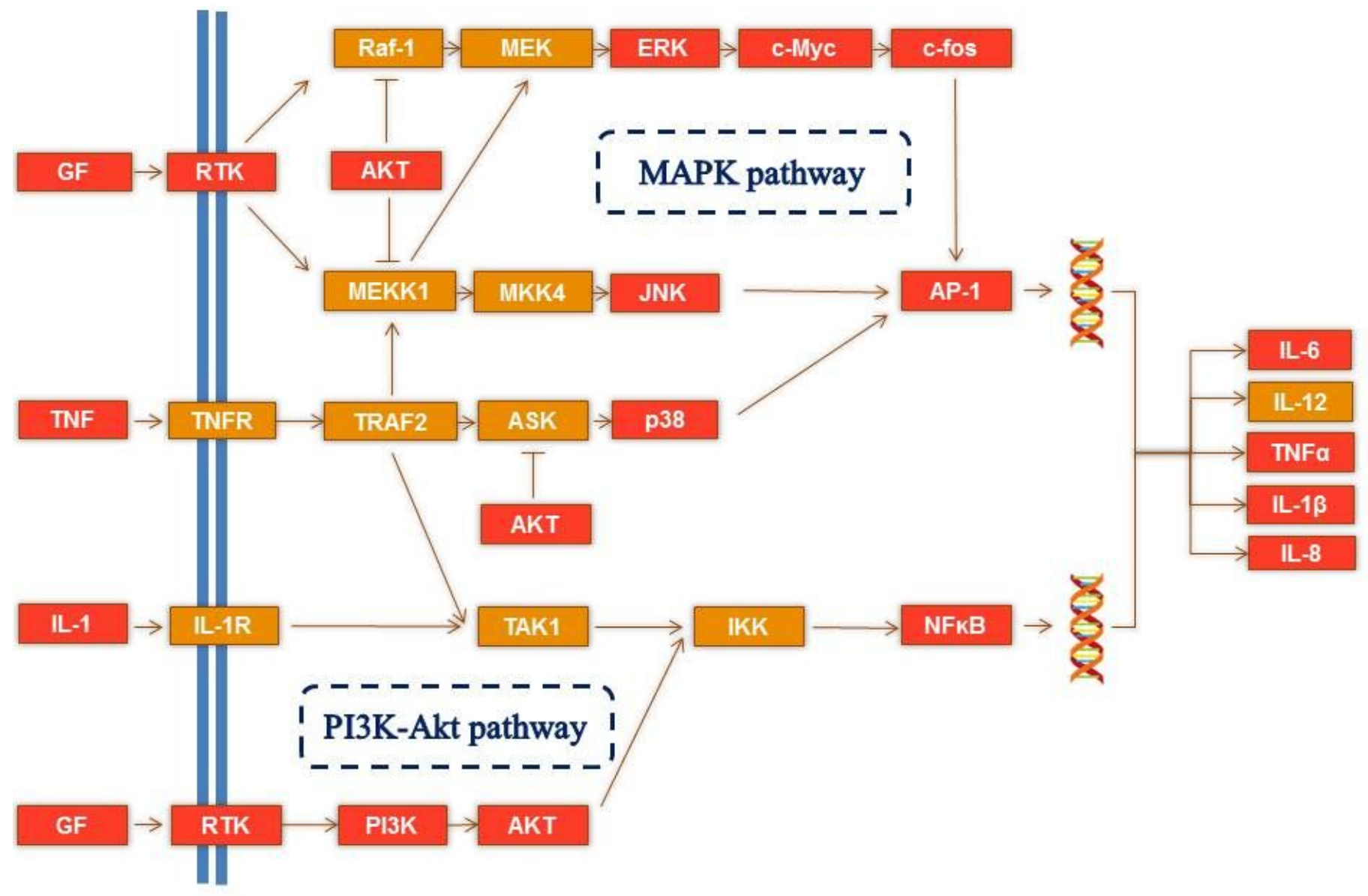

Figure 11

Distribution of the target proteins of ZJP on the predicted pathways (The red nodes are potential target proteins of ZJP, while the orange nodes are relevant targets in the pathway). 